\title{
AC 2008-1342: TEACHING REINFORCED CONCRETE DESIGN WITH MATHCAD APPLICATION
}

\section{Nirmal Das, Georgia Southern University}

Nirmal K. Das is an associate professor of Civil Engineering Technology at Georgia Southern University. He received a Bachelor of Civil Engineering degree from Jadavpur University, India, and M.S. and Ph.D. degrees in Civil Engineering (structures) from Texas Tech University. His areas of interest include structural analysis, structural reliability and wind engineering. Dr. Das is a registered professional engineer in Ohio and Georgia, and is a Fellow of the American Society of Civil Engineers. 


\title{
Teaching Reinforced Concrete Design with Mathcad Application
}

\begin{abstract}
Reinforced Concrete Design is typically offered as a senior-level required course in an undergraduate Civil Engineering or Civil Engineering Technology curriculum. The design of various components of a building structure is performed based on the American Concrete Institute (ACI) Building Code 318. One of the major topics covered in the course is design of beams. The design involves myriad computations to account for several aspects of the design: from determining the required strengths of the beam, to selecting an adequate beam size and the necessary amount of steel reinforcement, to checking for deflection and crack widths, to ensuring adequate development lengths for selected steel reinforcing bars. All these topics are usually covered as separate modules in traditional lecture-format instruction.

Typically code-based structural design uses trial-and-error procedure that often requires several iterations, involving tedious, repetitive calculations. Also, only a limited number of examples can be presented in the classroom due to time constraint. To circumvent the situation, a logical option is to capitalize on the computer's abilities to compute, and interface with the user.

The purpose of this paper is to present a computer-based problem-solving courseware that has been developed to complement traditional lecture-format delivery of the Reinforced Concrete Design course to enhance student learning. The courseware consists of several interlinked modules, each addressing a different aspect for design of a beam.. The Mathcad software, which supports custom designed documents called electronic books that explain complex topics and provide reusable computational templates, has been used to develop the modules. Besides allowing for faster solution of a problem, this tool is useful for providing live math examples that promote the exploration of the underlying relationships between problem variables and solutions. Thus, the students would have a holistic view of the entire design process; more specifically, they will have a better understanding of how the different design parameters are interconnected.
\end{abstract}

\section{Introduction}

The four-year ABET-accredited Civil Engineering Technology curriculum at Georgia Southern University includes a required, senior-level course in Reinforced Concrete Design. The two main objectives of the course are: (1) the students gain a thorough understanding of the fundamental principles underlying design of various structural components and the relevant stipulations in the ACI Code and (2) they correctly apply that knowledge to various practical design problems. A combination of homework and computational laboratory assignments is used for meeting the second objective. However, a significant amount of design activities is based on trial-and-error procedure that often requires several iterations, involving tedious, repetitive calculations. Also, only a limited number of examples can be presented in the classroom due to time constraint. To circumvent the situation, a logical option is to capitalize on the computer's abilities to compute, display graphics, and interface with the user, letting students focus on substantive issues and thereby engage in additional problem-solving for varied scenarios, outside the classroom at their own pace. 
Mathcad $^{l}$, an industry-standard calculation software, is used because it is as versatile and powerful as programming languages, yet it is as easy to learn as a spreadsheet. Additionally, it is linked to the Internet and other applications one uses everyday.

\section{Elements of Reinforced Concrete Design}

Concrete is basically an artificial or man-made stone made of a mixture of fine aggregate (sand) and coarse aggregate (crushed rock, or gravel), held together with a paste of cement and water. As with most rocks, concrete is very strong in compression, but very weak in tension. Reinforced concrete is a combination of concrete and steel wherein the steel reinforcement provides the tensile strength lacking in the concrete. Reinforced concrete is one of the most important materials available for construction.

A typical R.C. building frame consists of beams, columns and footings. These various reinforced concrete components are designed on the basis of the American Concrete Institute's Building Code Requirements for Structural Concrete (ACI 318) ${ }^{2}$. The ACI Code uses the strength design principle which can be stated as follows: the required strength of a component must not exceed the component's design strength. The required strength is determined through use of applicable loads and the corresponding load factors to account for overloading possibilities, as per the ACI Code. On the other hand, the design strength represents a limiting value of strength that is calculated including reductions for factors which may contribute to under-strength and the uncertainty of material properties.

Two types of problems are usually dealt with by the students in a Reinforced Concrete Design course: analysis and design. Whereas in an analysis problem, the load carrying capacity of a given component is determined, in a design problem, size of a component is determined to support a given or estimated loading. Three types of beams are commonly used in a reinforced concrete building: singly-reinforced rectangular beams, doubly-reinforced rectangular beams and T-beams. In this paper, only single-span, simply-supported and cantilever, singly-reinforced rectangular concrete beams are discussed.

Since a simply-supported beam carries only positive moment (tension in the bottom), steel reinforcement needs to be placed near the bottom of the beam. On the other hand, a cantilever beam carries only negative moment (tension at the top), and so it needs to be reinforced with steel bars placed near the top of the beam.

\section{Mathcad Program Features}

The program developed consists of five modules. The first module calculates the required strengths (moment and shear) for the two support types. The next two modules calculate the flexural reinforcement and shear reinforcement, respectively. The fourth module calculates beam deflection and the last one checks if the crack width is within an allowable limit. Detailed discussions on fundamentals of design of reinforced concrete structures can be found in any standard textbook ${ }^{3,4,5}$. 
The following information is required as input data: beam type (simply-supported and cantilever), beam length, magnitudes of distributed loads, magnitudes and locations of concentrated loads, load types (dead loads and live loads), material properties (compressive strength of concrete and yield strength of steel reinforcement), beam exposure type (dry, moist etc.), percentage of sustained live load, and duration of loading. The solution to a design problem obtained through use of the program consists of: beam size (width and overall thickness), amount of flexural reinforcement, amount of shear reinforcement (stirrups), magnitude of beam deflection (both instantaneous and long-term) and crack width for the beam under given loading.

The program developed using Mathcad is given in Appendix. Two example problems are presented next. . The first example is on design of a simply-supported rectangular beam, and the second example is on design of a cantilever beam.

\section{Example 1}

Design a singly-reinforced rectangular section for the simply-supported beam and loadings shown in Figure 1, considering moment and shear. All loads are service loads, and the dead load does not include the weight of the beam. Use compressive strength of concrete, $\mathrm{f}_{\mathrm{c}} \mathrm{c}=4000 \mathrm{psi}$ and yield strength of steel reinforcement (for both moment and shear), $f_{y}=60,000$ psi. Assume normal-weight concrete (unit weight $=150 \mathrm{pcf}$ ) to be used and the beam will be used as floor beam exposed to dry air. Include computations of both instantaneous deflection and long-term deflection after 4 years, assuming $30 \%$ of live load will be sustained. Also perform a check for crack width.

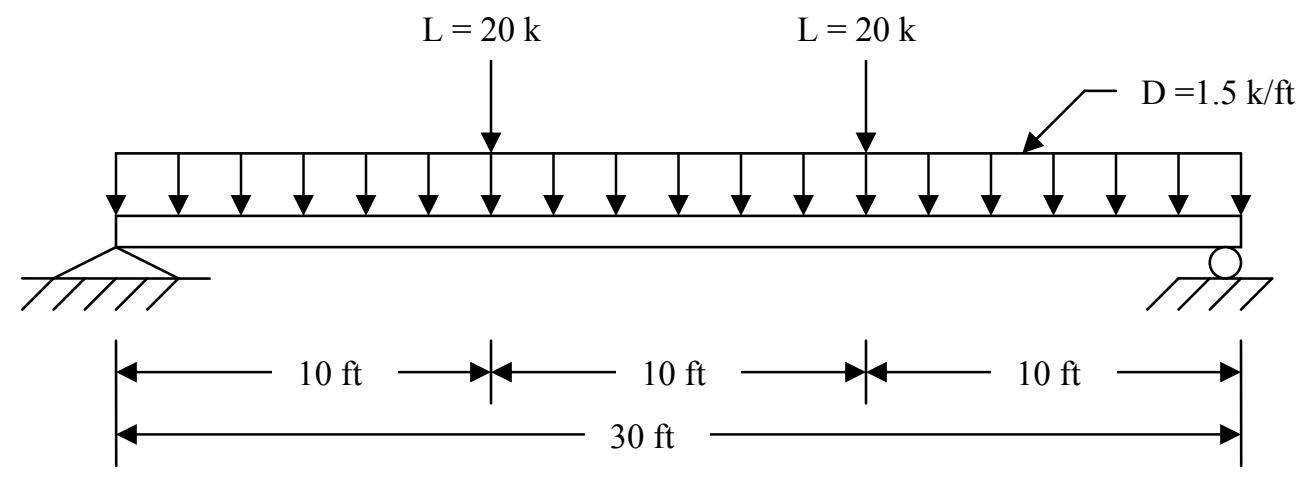

Figure 1

Input Data:

$$
\begin{array}{llll}
\text { Case }:=1 & \mathrm{n}:=3 & \mathrm{~L}:=30 \cdot \mathrm{ft} & \mathrm{kD}:=1 \quad \mathrm{~kL}:=2 \\
\mathrm{P}_{\mathrm{D}}:=\left(\begin{array}{lll}
0 & 0 & 0
\end{array}\right)^{\mathrm{T}} \cdot \mathrm{lb} & \mathrm{P}_{\mathrm{L}}:=\left(\begin{array}{llll}
20000 & 0 & 20000
\end{array}\right)^{\mathrm{T}} \cdot \mathrm{lb} \quad \mathrm{dist}:=\left(\begin{array}{lll}
10 & 15 & 20
\end{array}\right)^{\mathrm{T}} \cdot \mathrm{ft} \\
\mathrm{w}_{\mathrm{D}}:=1500 \frac{\mathrm{lb}}{\mathrm{ft}} & \mathrm{w}_{\mathrm{L}}:=0 \cdot \frac{\mathrm{lb}}{\mathrm{ft}} & \gamma:=150 \cdot \frac{\mathrm{lb}}{\mathrm{ft}^{3}}
\end{array}
$$


$\mathrm{d}_{\mathrm{c}}:=3 \cdot \mathrm{in} \quad \mathrm{f}_{\mathrm{c}}:=4000 \mathrm{psi} \quad \mathrm{f}_{\mathrm{y}}:=60000 \mathrm{psi}$

month $:=2592000 \mathrm{sec} \quad$ Duration $:=48 \cdot$ month $\quad$ Percent $:=30 \quad$ Exposure $:=$ "Dry air"

\section{Solution:}

\section{Required Shear and Moment Strengths:}

Shear and moment diagrams due to factored loads:
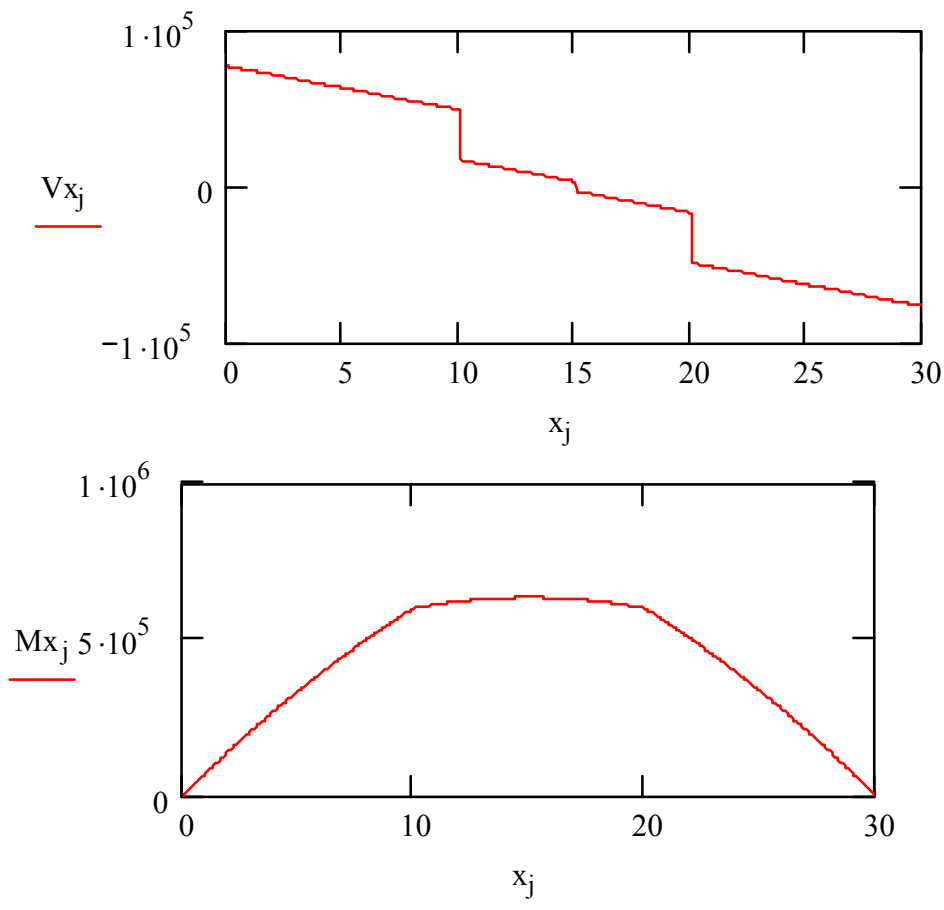

Required shear strength: $\quad V_{\mathrm{u}}=7.685 \times 10^{4} \mathrm{lbf}$

Required moment strength: $\quad \mathrm{M}_{\mathrm{u}}=6.339 \times 10^{5} \mathrm{ft} \cdot \mathrm{lbf}$

\section{Design for moment:}

Selected beam width: $\quad b_{\mathrm{w}}=22 \mathrm{in} \quad$ Selected beam overall thickness: $\mathrm{h}=36$ in

Required area of steel: $\mathrm{A}_{\mathrm{S}}=4.516 \mathrm{in}^{2} \quad$ Selected flexural reinforcement $=5 \# 9$ bars

\section{Design for Shear:}

Assuming 2-legged \#3 stirrups:

Actual stirrup spacing to be used: $\mathrm{s}_{\text {actual }}=12 \mathrm{in}$

\section{Check for Deflection:}

Instantaneous deflection due to service dead loads: $\quad \delta_{\mathrm{D}}=0.342$ in 
Instantaneous deflection due to service live loads: $\quad \delta_{\mathrm{L}}=0.271$ in

Instantaneous deflection due to dead plus live loads: $\delta_{\text {total }}=0.613$ in

Long-term deflection: $\quad \delta_{\mathrm{LT}}=1.106 \mathrm{in}$

\section{Check for Crack Width:}

Estimated crack width: $\quad \mathrm{w}_{\mathrm{cr}}=0.014$ in

Allowable crack width: $\mathrm{w}_{\text {allowable }}=0.016 \mathrm{in}$

crackwidth $=$ "OK"

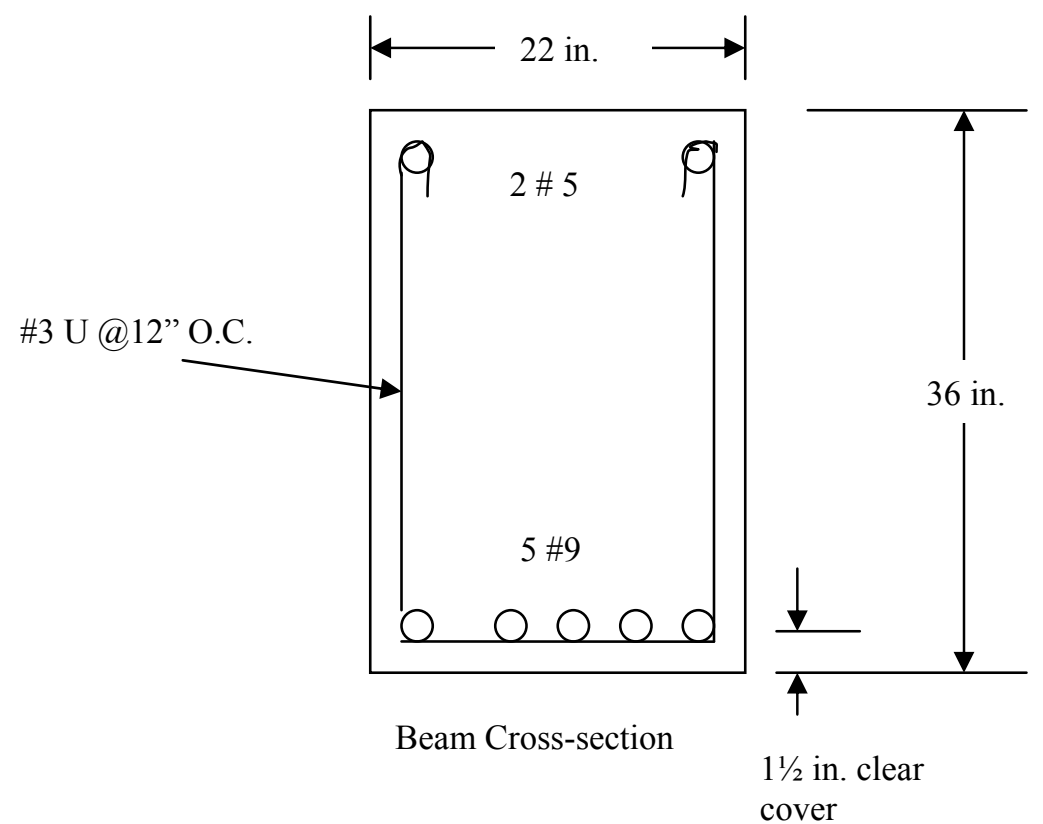

\section{Example 2}

Design a singly-reinforced rectangular section for the cantilever beam and loadings shown in Figure 2. All loads are service loads, and the dead load does not include the weight of the beam. Use compressive strength of concrete, $\mathrm{f}^{\prime}{ }_{\mathrm{c}}=4000 \mathrm{psi}$ and yield strength of steel reinforcement (for both moment and shear), $\mathrm{f}_{\mathrm{y}}=60,000 \mathrm{psi}$. Assume normal-weight concrete (unit weight $=150$ pcf) to be used and the beam will be used as floor beam exposed to dry air. Include computations of both instantaneous deflection and long-term deflection after 5 years, assuming $30 \%$ of live load will be sustained. Also perform a check for crack width. 


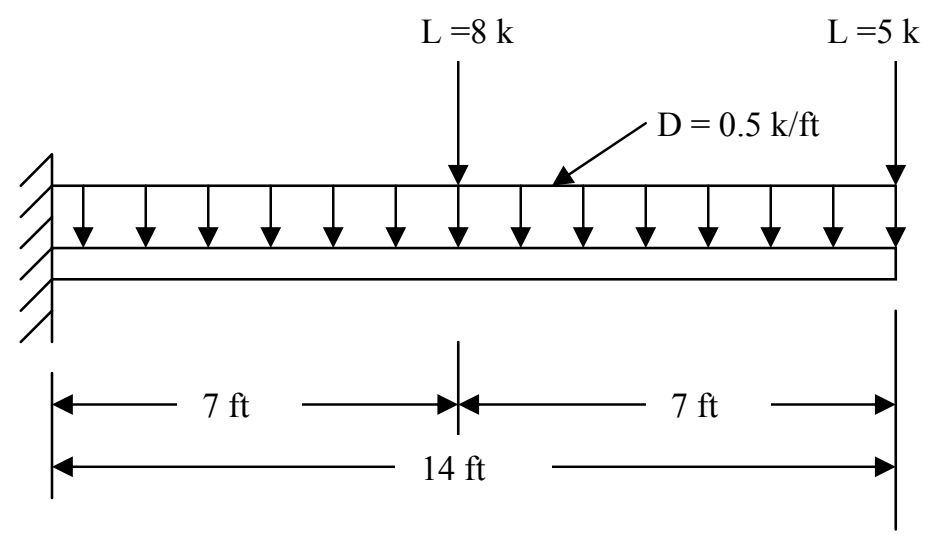

Figure 2

\section{Input Data:}

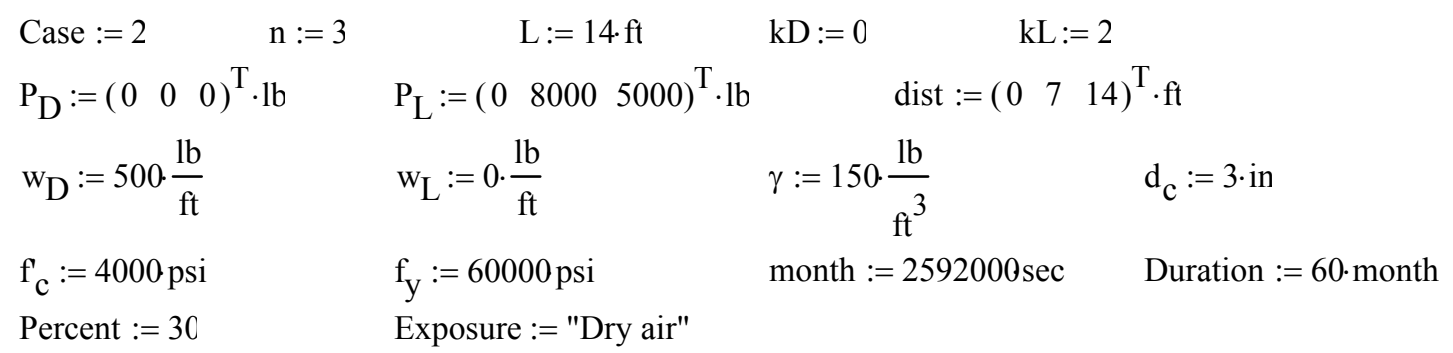

\section{Solution:}

\section{Required Shear and Moment Strengths:}

Shear and moment diagrams due to factored loads:

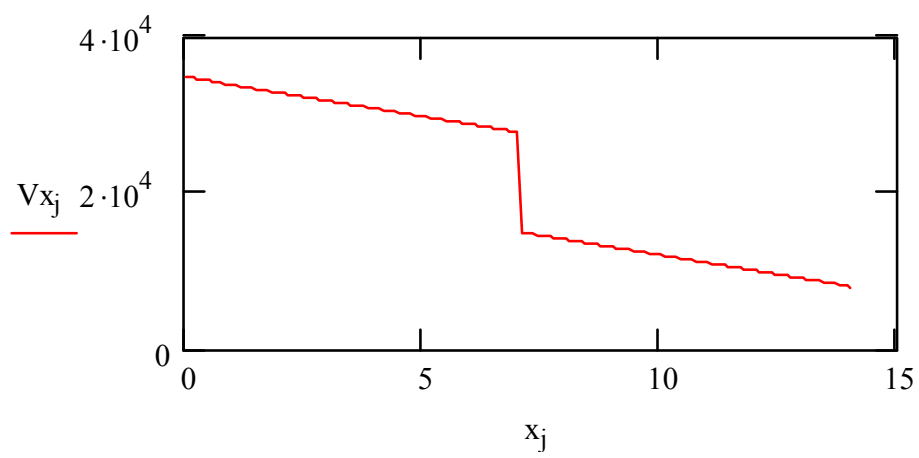




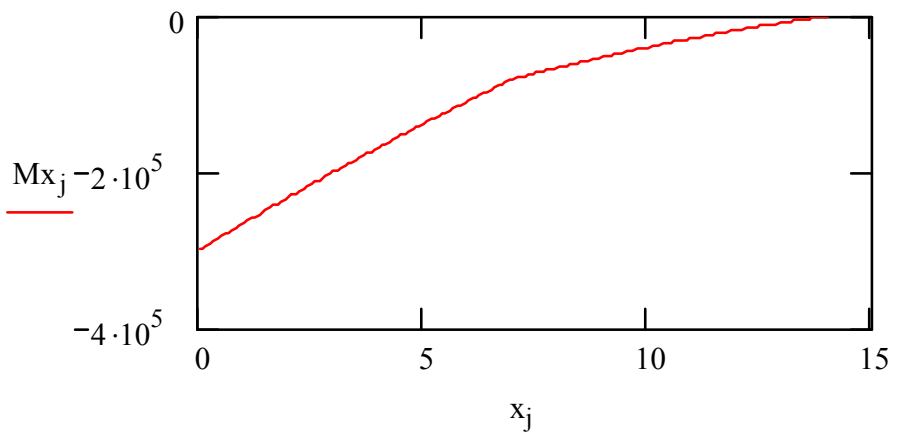

Required shear strength: $\mathrm{V}_{\mathrm{u}}=3.483 \times 10^{4} \mathrm{lbf}$

Required moment strength: $\mathrm{M}_{\mathrm{u}}=2.998 \times 10^{5} \mathrm{ft} \cdot \mathrm{lbf}$

\section{Design for moment:}

Selected beam width: $b_{w}=14$ in $\quad$ Selected beam overall thickness: $h=23$ in

Required area of steel: $\mathrm{A}_{\mathrm{S}}=3.782 \mathrm{in}^{2} \quad$ Selected flexural reinforcement $=4 \# 9$ bars

\section{Design for Shear:}

Assuming 2-legged \#3 stirrups:

Actual stirrup spacing to be used: $\mathrm{s}_{\text {actual }}=9$ in

\section{Check for Deflection:}

Instantaneous deflection due to service dead loads: $\delta_{\mathrm{D}}=0.028 \mathrm{in}$

Instantaneous deflection due to service live loads: $\delta_{\mathrm{L}}=0.152 \mathrm{in}$

Instantaneous deflection due to dead plus live loads: $\delta_{\text {total }}=0.179$ in

Long-term deflection due to dead load plus sustained live loads: $\delta_{\mathrm{LT}}=0.298 \mathrm{in}$

\section{Check for Crack Width:}

Estimated crack width: $\mathrm{w}_{\mathrm{cr}}=0.013$ in

Allowable crack width: $\mathrm{w}_{\text {allowable }}=0.016 \mathrm{in}$

crackwidth $=$ "OK" 


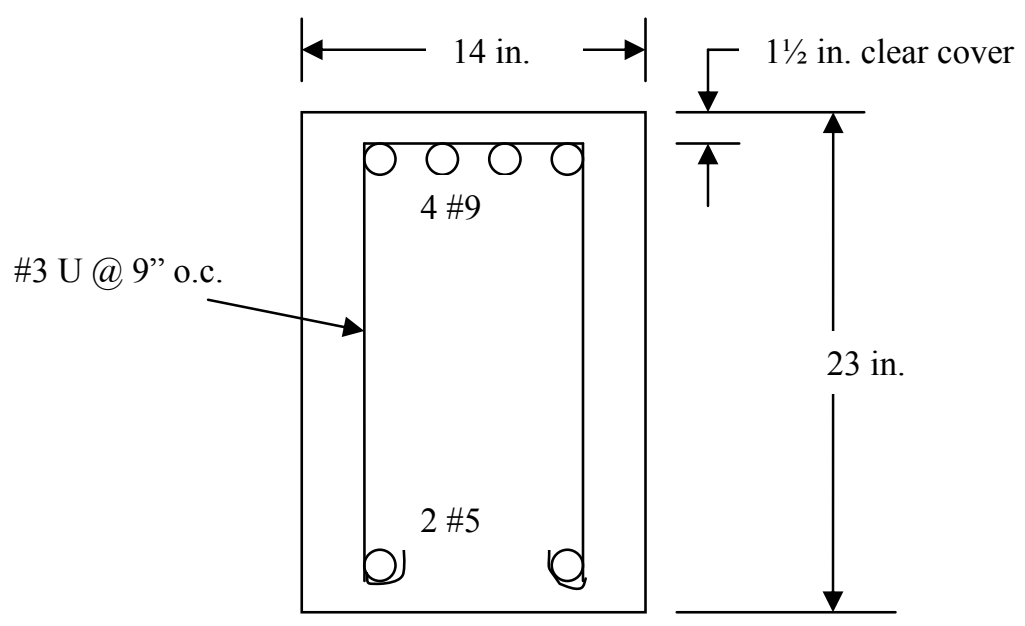

Beam cross-section

\section{Software Integration}

It is planned to incorporate this program into the Reinforced Concrete Design course in Fall 2008 semester. This is a 4-credit course that has a 2-hour computational laboratory component. Typically, laboratory assignments and group projects would require the use of this tool that is accessible in a dedicated computer laboratory. The author is aware of certain limitations in the program developed, and plans to assign tasks of modifying segments of the program as group exercises to the class.. Through these exercises, students would learn to write programs using Mathcad to find solutions to a variety of problems. Students are required to support their results with other manual calculations. If group projects are done using these tools, students are required to make presentations and share the experience with others in the class. It has been the author's experience that such in-class presentations not only help students sharpen their communication skills, but also demonstrate the benefits of using such software tools in the learning process. It is also planned to obtain and report student feedback on integration of this tool into the course. Additionally, the author plans to augment the courseware with additional modules.

\section{Summary}

Mathcad has been used as a problem-solving tool in Reinforced Concrete Design course. It provides a viable means for students to solve structural design problems using a user-friendly, yet versatile software. Students exposed to Mathcad and its capabilities in computation and visualization are expected to develop a better understanding of the materials presented in the course. Overall, the incorporation of Mathcad would likely result in a positive, efficient, and more effective teaching-learning environment.

\section{References}

1. Mathsoft, Mathcad 11 User's Guide, Mathsoft Engineering \& Education, Inc.

2. American Concrete Institute, Building Code Requirements for Structural Concrete (ACI 318-05) and Commentary (ACI 318R-05), An ACI Standard 
3. McCormac, J. C., Design of Reinforced Concrete, $7^{\text {th }}$ ed, John Wiley \& Sons, Inc..

4. Nawy, E. G., Reinforced Concrete-A Fundamental Approach, $4^{\text {th }}$ ed, Prentice Hall, Inc.

5. MacGregor, J. G., Reinforced Concrete- Mechanics and Design, 3rd ed, Prentice Hall, Inc.

\section{Appendix}

\section{Mathcad Program for Design of Rectangular Singly-Reinforced Concrete Beams}

\section{Input Variables:}

Case $\quad$ Support type $(1=$ Simply-supported, 2 = Cantilever $)$

L Beam span

$\mathrm{kD} \quad$ Number of concentrated dead loads

$\mathrm{kL} \quad$ Number of concentrated live loads

$\mathrm{P}_{\mathrm{D}, \mathrm{i}} \quad$ I-th concentrated service dead load

$\mathrm{P}_{\mathrm{L}, \mathrm{i}} \quad$ I-th concentrated service live load

n Number of concentrated load points

dist $_{i} \quad$ Distance to $\mathrm{i}$-th concentrated load, from the left end

${ }^{\mathrm{w}} \mathrm{D} \quad$ Uniformly distributed service dead load (not including beam weight)

$\mathrm{w}_{\mathrm{L}} \quad$ Uniformly distributed service live load

$\mathrm{f}_{\mathrm{c}} \quad$ Compressive strength of concrete

$f_{y} \quad$ Yield strength of steel reinforcement

Duration Duration of sustained loads (months)

Percent Percentage of live load considered as sustained load

Exposure Beam exposure (dry air, moist air etc.)

$\mathrm{d}_{\mathrm{c}} \quad$ Effective cover

$\ddot{i} \quad$ Unit weight of concrete

\section{Required Shear and Moment Strengths:}

Factored loads (concentrated):

$\mathrm{i}:=1 . . \mathrm{n}$

$\mathrm{P}_{\mathrm{U}_{\mathrm{i}}}:=1.2 \cdot \mathrm{P}_{\mathrm{D}_{\mathrm{i}}}+1.6 \cdot \mathrm{P}_{\mathrm{L}_{\mathrm{i}}}$

Factored loads (distributed):

$\mathrm{h}_{1}:=\mid \begin{array}{ll}\frac{\mathrm{L}}{10} & \text { if Case }=1 \\ \frac{\mathrm{L}}{7.5} & \text { if } \text { Case }=2\end{array}$

Assumed overall thickness: $\mathrm{h}_{2}:=\mathrm{h}_{1} \cdot \frac{12}{\operatorname{UnitsOf}\left(\mathrm{h}_{1}\right)} \quad \mathrm{h}:=\operatorname{ceil}\left(\mathrm{h}_{2}\right) \cdot$ in

Assumed width: $\mathrm{b}_{1}:=0.6 \cdot \mathrm{h} \quad \mathrm{b}_{2}:=\mathrm{b}_{1} \cdot \frac{12}{\operatorname{UnitsOf}\left(\mathrm{b}_{1}\right)} \quad \mathrm{b}:=\operatorname{ceil}\left(\mathrm{b}_{2}\right)$ in

Assumed beam weight: $\mathrm{w}_{\text {beam }}:=\mathrm{b} \cdot \mathrm{h} \cdot \gamma \quad \mathrm{w}_{\mathrm{U}}:=1.2 \cdot\left(\mathrm{w}_{\mathrm{D}}+\mathrm{w}_{\text {beam }}\right)+1.6 \cdot \mathrm{w}_{\mathrm{L}}$

Provide plotting information: 
Number of divisions in the beam length for plots of shear and moment diagrams: div $:=\frac{\mathrm{L}}{\operatorname{UnitsOf}(\mathrm{L})} \cdot 12$

Number of points: pts $:=\operatorname{div}+1 \quad$ Interval between points: int $:=\frac{\mathrm{L}}{\operatorname{div}}$

Factored Shear and moment:

(a) Due to factored concentrated loads:

$\mathrm{i}:=1$.. n

Left support reaction:

$\operatorname{Ayp}_{\mathrm{i}}:=\left.\right|_{\mathrm{U}_{\mathrm{i}}} \cdot\left[1-\left(\frac{\mathrm{dist}_{\mathrm{i}}}{\mathrm{L}}\right)\right]$ if Case $=1$
$\mathrm{P}_{\mathrm{U}_{\mathrm{i}}}$ if Case $=2$
$\mathrm{j}:=1 . . \mathrm{pts}$
$\mathrm{x}_{\mathrm{j}}:=(\mathrm{j}-1) \cdot$ int

Shear at point $\mathrm{x}_{\mathrm{j}}$ due to concentrated load $\mathrm{P}_{\mathrm{U}_{\mathrm{i}}}$ :

$\mathrm{Vpx}_{1, j}:=\mid \begin{aligned} & \operatorname{Ayp}_{\mathrm{i}} \text { if } \mathrm{x}_{\mathrm{j}} \leq \mathrm{dist}_{\mathrm{i}} \\ & \operatorname{Ayp}_{\mathrm{i}}-\mathrm{P}_{\mathrm{U}_{\mathrm{i}}} \text { otherwise }\end{aligned}$

Moment at point $\mathrm{x}_{\mathrm{j}}$ due to concentrated load $\mathrm{P}_{\mathrm{U}_{\mathrm{j}}}$ :

$\operatorname{Mpx}_{i, j}:=\left[\left(\begin{array}{l}\operatorname{Ayp}_{i} \cdot x_{j} \text { if }(\text { Case }=1) \wedge\left(x_{j} \leq \text { dist }_{i}\right) \\ \operatorname{Ayp}_{i} \cdot x_{j}-P_{U_{i}} \cdot\left(x_{j}-\operatorname{dist}_{i}\right) \text { if }(\text { Case }=1) \wedge\left(x_{j}>\operatorname{dist}_{i}\right) \\ \left(\operatorname{Ayp}_{i} \cdot x_{j}\right)-\left(\operatorname{Ayp}_{i} \cdot \operatorname{dist}_{i}\right) \text { if }(\text { Case }=2) \wedge\left(x_{j} \leq \operatorname{dist}_{i}\right) \\ 0 \text { if }(\text { Case }=2) \wedge\left(x_{j}>\text { dist }_{i}\right)\end{array}\right]\right.$

(b) Due to factored distributed loads:

Left support reaction:

Ayw $:=\mid \begin{aligned} & 0.5 \cdot \mathrm{w}_{\mathrm{U}} \cdot \mathrm{L} \text { if Case }=1 \\ & \mathrm{w}_{\mathrm{U}} \cdot \mathrm{L} \text { if Case }=2\end{aligned}$

$\mathrm{j}:=1$..pts

$\mathrm{x}_{\mathrm{j}}:=(\mathrm{j}-1) \cdot \mathrm{int}$

Shear at point $\mathrm{x}_{\mathrm{j}}$ due to distributed load $\mathrm{w}_{\mathrm{U}}: \mathrm{Vwx}_{\mathrm{j}}:=\left(\right.$ Ayw $\left.-\mathrm{w}_{\mathrm{U}} \cdot \mathrm{x}_{\mathrm{j}}\right)$

Moment at point $\mathrm{x}_{\mathrm{j}}$ due to distributed load $\mathrm{w}_{\mathrm{U}}$ :

$\operatorname{Mwx}_{\mathrm{j}}:=\mid \begin{aligned} & \text { Ayw } \cdot \mathrm{x}_{\mathrm{j}}-\mathrm{w}_{\mathrm{U}} \cdot \frac{\left(\mathrm{x}_{\mathrm{j}}\right)^{2}}{2} \text { if Case }=1 \\ & \text { Ayw } \cdot \mathrm{x}_{\mathrm{j}}-\mathrm{w}_{U} \cdot \frac{\left(\mathrm{x}_{\mathrm{j}}\right)^{2}}{2}-\frac{\mathrm{w}_{\mathrm{U}} \cdot \mathrm{L}^{2}}{2} \text { if Case }=2\end{aligned}$

(c) Due to all factored loads (concentrated and distributed):

$\mathrm{j}:=1$.. pts

Shear at $\mathrm{x}_{\mathrm{j}}$ : 
$\mathrm{Vx}_{\mathrm{j}}:=\left(\sum_{\mathrm{i}=1}^{\mathrm{n}} \mathrm{Vpx}_{1, \mathrm{j}}\right)+\mathrm{Vwx}_{\mathrm{j}}$

Moment at $x_{j}$ :

$M x_{j}:=\left(\sum_{i=1}^{n} M x_{i, j}\right)+M w x_{j}$

(d) From shear and moment diagrams due to factored loads:

Required shear strength: $\mathrm{V}_{\mathrm{u}}:=\max (\mathrm{Vx}) \cdot \mathrm{g}$

Required moment strength:

$\mathrm{M}_{\mathrm{u} 1}:=\mid \begin{array}{ll}\max (\mathrm{Mx}) \cdot \mathrm{g} & \text { if Case }=1 \\ \min (\mathrm{Mx}) \cdot \mathrm{g} & \text { if Case }=2\end{array}$

$\mathrm{M}_{\mathrm{u}}:=\left|\mathrm{M}_{\mathrm{u} 1}\right|$

\section{Design for moment:}

Effective depth of beam: $d:=h-d_{c}$

Moment capacity reduction factor: $\phi:=0 . \mathcal{S}$ (Assumed)

Nominal moment strength required of beam cross-section: $M_{n}:=\frac{M_{u}}{\phi}$

Coefficient of resistance: $R_{n}:=\frac{M_{n}}{b \cdot d^{2}}$

Required steel percentage :

$\rho_{\text {reqd }}:=0.85 \cdot \frac{\mathrm{f}_{\mathrm{c}}}{\mathrm{f}_{\mathrm{y}}} \cdot\left(1-\sqrt{1-\frac{2 \cdot \mathrm{R}_{\mathrm{n}}}{0.85 \cdot \mathrm{f}_{\mathrm{c}}^{\prime}}}\right)$

Check for minimum percentage of steel reinforcement:

$\rho_{\min 1}:=\frac{200 \cdot \mathrm{psi}}{\mathrm{f}_{\mathrm{y}}}$

$\rho_{\min 2}:=3 \cdot\left(\frac{\sqrt{\mathrm{f}_{\mathrm{c}}}}{\mathrm{f}_{\mathrm{y}}}\right) \cdot \sqrt{\mathrm{psi}}$

$\rho_{\min }:=\mid \begin{array}{ll}\rho_{\min 1} & \text { if } \rho_{\min 1}>\rho_{\min 2} \\ \rho_{\min 2} & \text { otherwise }\end{array}$

Check for maximum percentage of steel reinforcement:

A minimum strain of 0.005 is required for tensile reinforcement to ensure ductile behavior of beam. The depth of actual neutral axis for that condition which corresponds to maximum tensile steel permitted by the ACI Code:

$\mathrm{c}:=\left(\frac{0.003}{0.003+0.005}\right) \cdot \mathrm{d}$

(Note: Strain in concrete at ultimate stage $=0.003$ )

Coefficient value: 


$$
\beta_{1}:=\mid \begin{aligned}
& 0.85 \text { if } \mathrm{f}_{\mathrm{c}} \leq 4000 \mathrm{psi} \\
& 0.85-\frac{\left(\mathrm{f}_{\mathrm{c}}-4000 \mathrm{psi}\right) \cdot 0.05}{1000 \mathrm{psi}} \text { if } \mathrm{f}_{\mathrm{c}}>4000 \mathrm{psi} \\
& 0.65 \text { if }\left[0.85-\frac{\left(\mathrm{f}_{\mathrm{c}}-4000 \mathrm{psi}\right) \cdot 0.05}{1000 \mathrm{psi}}\right] \leq 0.65
\end{aligned}
$$

Depth of Whitney's stress block:

$\mathrm{a}:=\beta_{1} \cdot \mathrm{c}$

Maximum percentage of steel:

$\rho_{\max }:=\frac{\mathrm{a}}{\mathrm{d}} \cdot\left(0.85 \cdot \frac{\mathrm{f}_{\mathrm{c}}}{\mathrm{f}_{\mathrm{y}}}\right)$

$\rho:=\mid \rho_{\text {min }}$ if $\rho_{\text {reqd }}<\rho_{\text {min }}$

$\rho_{\text {reqd }}$ if $\left(\rho_{\text {reqd }} \geq \rho_{\text {min }}\right) \wedge\left(\rho_{\text {reqd }} \leq \rho_{\text {max }}\right)$

"Beyond maximum permitted; use bigger beam section" otherwise

$\phi:=0.9$ if $\rho \leq \rho_{\max }$

"Less than 0.9" otherwise

Required area of steel:

$A_{S}:=\rho \cdot b \cdot d$

Bars :=

\begin{tabular}{|r|r|r|}
\hline & 1 & 2 \\
\hline 1 & 3 & 0.38 \\
\hline 2 & 4 & 0.5 \\
\hline 3 & 5 & 0.63 \\
\hline 4 & 6 & 0.75 \\
\hline 5 & 7 & 0.88 \\
\hline 6 & 8 & 1 \\
\hline 7 & 9 & 1.13 \\
\hline 8 & 10 & 1.27 \\
\hline 9 & 11 & 1.41 \\
\hline
\end{tabular}

Note: Column 1: Bar number

Column 2: Bar diameter (inches)

Assuming \#9 bars, bar diameter: $d_{b}:=1.128$ in $\quad$ Area of bar: $A_{b}:=\pi \frac{d_{b}{ }^{2}}{4}$

Number of $\# 9$ bars required: num $:=\frac{\mathrm{A}_{\mathrm{s}}}{\mathrm{A}_{\mathrm{b}}} \quad \mathrm{m}:=\operatorname{ceil}(\mathrm{num})$

Minimum width required: $b_{\min }:=m \cdot d_{b}+(m-1) \cdot d_{b}+2 \cdot(2.5 \cdot$ in $)$

Selected beam width:

$\mathrm{b}_{\mathrm{w}}:=\| \begin{aligned} & \mathrm{b} \text { if } \mathrm{b} \geq \mathrm{b}_{\min } \\ & \text { ceil }\left(\mathrm{b}_{\text {min }}\right) \cdot \text { in otherwise }\end{aligned}$

reinforcement $:=\mathrm{m}$ 


\section{Design for Shear:}

Shear capacity reduction factor: $\phi_{\mathrm{V}}:=0.7 \xi$

Nominal (theoretical) shear strength of concrete: $\mathrm{V}_{\mathrm{c}}:=2 \cdot \sqrt{\mathrm{f}_{\mathrm{c}}} \cdot \mathrm{b} \cdot \mathrm{d} \cdot \sqrt{\mathrm{psi}}$

Nominal (theoretical) shear strength of stirrups:

$\mathrm{V}_{\mathrm{s}}:=\frac{\left(\mathrm{V}_{\mathrm{u}}-\phi_{\mathrm{v}} \cdot \mathrm{V}_{\mathrm{c}}\right)}{\phi_{\mathrm{v}}}$

Stirrups := "Not required" if $\mathrm{V}_{\mathrm{u}}<0.5 \cdot\left(\phi_{\mathrm{v}} \cdot \mathrm{V}_{\mathrm{c}}\right)$

"Minimum amount required" if $\left(\mathrm{V}_{\mathrm{u}} \leq \phi_{\mathrm{V}} \cdot \mathrm{V}_{\mathrm{c}}\right) \wedge\left(\mathrm{V}_{\mathrm{u}} \geq 0.5 \cdot \phi_{\mathrm{V}} \cdot \mathrm{V}_{\mathrm{c}}\right)$

"Required--calculations shown below" if $\left(\mathrm{V}_{\mathrm{s}} \leq 2 \cdot \mathrm{V}_{\mathrm{c}}\right)$

"Required--calculations shown below" if $\left(\mathrm{V}_{\mathrm{s}} \leq 4 \cdot \mathrm{V}_{\mathrm{c}}\right) \wedge\left(\mathrm{V}_{\mathrm{s}}>2 \cdot \mathrm{V}_{\mathrm{c}}\right)$

"Bigger beam section needed--revise design" if $\mathrm{V}_{\mathrm{s}}>4 \cdot \mathrm{V}_{\mathrm{c}}$

Stirrups = "Required--calculations shown below"

Assuming 2-legged \#3 stirrups:

Diameter of stirrup: $\mathrm{d}_{\text {stirrup }}:=0.375$ in

Cross-sectional area of stirrup: $\mathrm{A}_{\mathrm{V}}:=2 \cdot\left(\pi \frac{\mathrm{d}_{\text {stirrup }}}{4}\right)$

Required stirrup spacing: $\mathrm{s}_{\text {reqd }}:=\mathrm{A}_{\mathrm{v}} \cdot \mathrm{f}_{\mathrm{y}} \cdot \frac{\mathrm{d}}{\mathrm{V}_{\mathrm{S}}}$

Maximum allowable spacing based on shear reinforcement requirement:

$\mathrm{s}_{\max 1}:=\mid 0.5 \cdot \mathrm{d}$ if $(0.5 \cdot \mathrm{d}<24 \cdot \mathrm{in}) \wedge\left(\mathrm{V}_{\mathrm{s}} \leq 2 \cdot \mathrm{V}_{\mathrm{c}}\right)$

24. in if $(0.5 \cdot \mathrm{d} \geq 24 \cdot$ in $) \wedge\left(\mathrm{V}_{\mathrm{s}} \leq 2 \cdot \mathrm{V}_{\mathrm{c}}\right)$

$0.25 \cdot \mathrm{d}$ if $(0.25 \cdot \mathrm{d}<12 \cdot \mathrm{in}) \wedge\left(\mathrm{V}_{\mathrm{s}}>2 \cdot \mathrm{V}_{\mathrm{c}}\right)$

$12 \cdot$ in if $(0.25 \cdot \mathrm{d} \geq 12 \cdot \mathrm{in}) \wedge\left(\mathrm{V}_{\mathrm{s}}>2 \cdot \mathrm{V}_{\mathrm{c}}\right)$

Maximum allowable spacing to provide minimum area of shear reinforcement: $s_{1}:=\frac{\left(\mathrm{A}_{\mathrm{v}} \cdot \mathrm{f}_{\mathrm{y}}\right)}{0.75 \cdot \sqrt{\mathrm{f}_{\mathrm{c}}} \cdot \mathrm{b}_{\mathrm{w}} \cdot \sqrt{\mathrm{psi}}}$

$$
\begin{aligned}
& \mathrm{s}_{2}:=\frac{\left(\mathrm{A}_{\mathrm{v}} \cdot \mathrm{f}_{\mathrm{y}}\right)}{50 \cdot \mathrm{psi} \cdot \mathrm{b}_{\mathrm{w}}} \\
& \mathrm{s}_{\max 2}:=\mid \begin{array}{ll}
\mathrm{s}_{1} \text { if } \mathrm{s}_{1}<\mathrm{s}_{2} \\
\mathrm{~s}_{2} \text { otherwise }
\end{array}
\end{aligned}
$$

Maximum allowable spacing for stirrups:

$\mathrm{s}_{\max }:=\mid \begin{aligned} & \mathrm{s}_{\max 1} \text { if } \mathrm{s}_{\max 1} \leq \mathrm{s}_{\max 2} \\ & \mathrm{~s}_{\max 2} \text { otherwise }\end{aligned}$

Spacing requirement:

$\mathrm{s}:=\| \mathrm{s}_{\text {reqd }}$ if $\mathrm{s}_{\text {reqd }} \leq \mathrm{s}_{\max }$ 
$\mathrm{s}_{\mathrm{a}}:=\frac{\mathrm{s} \cdot 12}{\text { UnitsOf(s) }}$

Actual stirrup spacing to be used: $\mathrm{s}_{\text {actual }}:=$ floor $\left(\mathrm{s}_{\mathrm{a}}\right) \cdot$ in

\section{Check for Deflection:}

Gross moment of inertia: $I_{g}:=b_{w} \cdot \frac{h^{3}}{12}$

Modulus of rupture: $\mathrm{f}_{\mathrm{r}}:=7.5 \cdot \sqrt{\mathrm{f}_{\mathrm{c}}} \cdot \sqrt{\mathrm{psi}}$

Cracking moment: $M_{c r}:=f_{r} \cdot \frac{I_{g}}{0.5 \cdot h}$

Modulus of elasticity of concrete: $E_{c}:=57000\left(\sqrt{f_{c}} \cdot \sqrt{p s i}\right)$

Modulus of elasticity of steel: $\mathrm{E}_{\mathrm{S}}:=29000000 \mathrm{psi}$

Modular ratio: $M R:=$ floor $\left(\frac{E_{S}}{E_{c}}\right)$

Locate neutral axis (equating moments of tensile and compressive areas about the neutral axis)::

$\mathrm{A}:=0.5 \cdot \mathrm{b}_{\mathrm{w}} \quad \mathrm{B}:=\mathrm{n} \cdot \mathrm{A}_{\mathrm{b}} \cdot \mathrm{m} \quad \mathrm{C}:=-\mathrm{B} \cdot \mathrm{d}$

$\mathrm{x}:=\frac{\left(-\mathrm{B}+\sqrt{\mathrm{B}^{2}-4 \cdot \mathrm{A} \cdot \mathrm{C}}\right)}{2 \cdot \mathrm{A}}$

Moment of inertia of cracked cross-section: $I_{c r}:=\frac{b_{w} \cdot x^{3}}{3}+M R \cdot A_{b} \cdot m \cdot(d-x)^{2}$

Moment due to service dead loads:

(a) Concentrated dead loads (only symmetric loads considered for Case 1)

$\mathrm{PD}:=\| \mathrm{i} \leftarrow 1$

while $\mathrm{i} \leq \mathrm{n}$

$\mid \begin{aligned} & \mathrm{P} \leftarrow \mathrm{P}_{\mathrm{D}_{\mathrm{i}}} \\ & \text { break if } \mathrm{P}>0 \\ & \mathrm{i} \leftarrow \mathrm{i}+1\end{aligned}$

$\mathrm{M}_{\mathrm{DP}}:=\mid \begin{aligned} & 0 \cdot \mathrm{lb} \cdot \mathrm{ft} \text { if } \mathrm{kD}=0 \\ & 0.25 \cdot \mathrm{PD} \cdot \mathrm{L} \text { if }(\text { Case }=1) \wedge(\mathrm{kD}=1) \\ & 0.333 \mathrm{PD} \cdot \mathrm{L} \text { if }(\text { Case }=1) \wedge(\mathrm{kD}=2) \\ & 0.5 \cdot \mathrm{PD} \cdot \mathrm{L} \text { if }(\text { Case }=1) \wedge(\mathrm{kD}=3) \\ & \sum_{\mathrm{i}=1}^{\mathrm{n}}\left(\mathrm{P}_{\mathrm{D}_{\mathrm{i}}} \cdot \text { dist }_{\mathrm{i}}\right) \text { if }(\text { Case }=2) \wedge(\mathrm{kD} \neq 0)\end{aligned}$

(b) Distributed dead loads: 
$\mathrm{M}_{\mathrm{DL}}:=\frac{\left(\mathrm{w}_{\mathrm{D}}+\mathrm{w}_{\text {beam }}\right) \cdot \mathrm{L}^{2}}{8}$

Moment due to service live loads:

(a) Concentrated live loads (only symmetric loads considered for Case 1) :

$\mathrm{PL}:=\mid \begin{aligned} & \mathrm{i} \leftarrow 1 \\ & \text { while } \mathrm{i} \leq \mathrm{n} \\ & \mid \begin{array}{l}\mathrm{P} \leftarrow \mathrm{P}_{\mathrm{L}_{\mathrm{i}}} \\ \text { break if } \mathrm{P}>0 \\ \mathrm{i} \leftarrow \mathrm{i}+1\end{array}\end{aligned}$

$\mathrm{M}_{\mathrm{LP}}:=\mid \begin{aligned} & 0 \cdot \mathrm{lb} \cdot \mathrm{ft} \text { if } \mathrm{kL}=0 \\ & 0.25 \cdot \mathrm{PL} \cdot \mathrm{L} \text { if }(\text { Case }=1) \wedge(\mathrm{kL}=1) \\ & 0.333 \mathrm{PL} \cdot \mathrm{L} \text { if }(\text { Case }=1) \wedge(\mathrm{kL}=2) \\ & 0.5 \cdot \mathrm{PL} \cdot \mathrm{L} \text { if }(\text { Case }=1) \wedge(\mathrm{kL}=3) \\ & \sum_{\mathrm{i}=1}^{\mathrm{n}}\left(\mathrm{P}_{\mathrm{L}_{\mathrm{i}}} \cdot \text { dist }_{\mathrm{i}}\right) \text { if }(\text { Case }=2) \wedge(\mathrm{kL} \neq 0)\end{aligned}$

(b) Distributed live loads:

$\mathrm{M}_{\mathrm{LL}}:=\frac{\mathrm{w}_{\mathrm{L}} \cdot \mathrm{L}^{2}}{8}$

Service load moment due to dead load plus live load:

$\mathrm{M}_{\mathrm{a}}:=\left(\mathrm{M}_{\mathrm{DP}}+\mathrm{M}_{\mathrm{DL}}+\mathrm{M}_{\mathrm{LP}}+\mathrm{M}_{\mathrm{LL}}\right) \cdot \mathrm{g}$

Effective moment of inertia:

$\mathrm{I}_{\mathrm{e}}:=\left(\frac{\mathrm{M}_{\mathrm{cr}}}{\mathrm{M}_{\mathrm{a}}}\right)^{3} \cdot \mathrm{I}_{\mathrm{g}}+\left[1-\left(\frac{\mathrm{M}_{\mathrm{cr}}}{\mathrm{M}_{\mathrm{a}}}\right)^{3}\right] \cdot \mathrm{I}_{\mathrm{cr}}$

Instantaneous deflection:

Due to concentrated dead loads:

$$
\begin{aligned}
& \delta \mathrm{D}:=\frac{\mathrm{PD} \cdot \mathrm{L}^{3}}{\mathrm{E}_{\mathrm{c}} \cdot \mathrm{I}_{\mathrm{e}}} \cdot \mathrm{g} \\
& \delta_{\mathrm{DPi}}:=\mid \begin{array}{l}
0 \cdot \text { in } \text { if } \mathrm{kD}=0 \\
0.021 \cdot \delta \mathrm{D} \text { if }(\text { Case }=1) \wedge(\mathrm{kD}=1) \\
0.036 \delta \mathrm{D} \text { if }(\text { Case }=1) \wedge(\mathrm{kD}=2) \\
0.05 \cdot \delta \mathrm{D} \text { if }(\text { Case }=1) \wedge(\mathrm{kD}=3) \\
\frac{\mathrm{g}}{\left(6 \cdot \mathrm{E}_{\mathrm{c}} \cdot \mathrm{I}_{\mathrm{e}}\right)} \cdot \sum_{\mathrm{i}=1}^{\mathrm{n}}\left[\mathrm{P}_{\mathrm{D}_{\mathrm{i}}} \cdot\left(\mathrm{L}-\text { dist }_{\mathrm{i}}\right)^{2} \cdot\left(3 \cdot \mathrm{L}-\text { dist }_{\mathrm{i}}\right)\right] \text { if }(\text { Case }=2) \wedge(\mathrm{kD} \neq 0)
\end{array}
\end{aligned}
$$


Due to distributed dead loads: $\delta_{\text {Dwi }}:=\frac{5}{384} \cdot \frac{\left(\mathrm{w}_{\mathrm{D}}+\mathrm{w}_{\text {beam }}\right) \cdot \mathrm{L}^{4} \cdot \mathrm{g}}{\mathrm{E}_{\mathrm{c}} \cdot \mathrm{I}_{\mathrm{e}}}$

Instantaneous deflection due to service dead loads: $\delta_{\mathrm{D}}:=\delta_{\mathrm{DPi}}+\delta_{\mathrm{Dw}}$

Due to concentrated live loads: $\delta \mathrm{L}:=\frac{\mathrm{PL} \cdot \mathrm{L}^{3}}{\mathrm{E}_{\mathrm{c}} \cdot \mathrm{I}_{\mathrm{e}}} \cdot \mathrm{g}$

$$
\delta_{\mathrm{LPi}}:=\mid \begin{aligned}
& 0 \cdot \text { in } \text { if } \mathrm{kL}=0 \\
& 0.021 \cdot \delta \mathrm{L} \text { if }(\text { Case }=1) \wedge(\mathrm{kL}=1) \\
& 0.036 \delta \mathrm{L} \text { if }(\text { Case }=1) \wedge(\mathrm{kL}=2) \\
& 0.05 \cdot \delta \mathrm{L} \text { if }(\text { Case }=1) \wedge(\mathrm{kL}=3) \\
& \frac{\mathrm{g}}{\left(6 \cdot \mathrm{E}_{\mathrm{c}} \cdot \mathrm{I}_{\mathrm{e}}\right)} \cdot \sum_{\mathrm{i}=1}^{\mathrm{n}} \mathrm{P}_{\mathrm{L}_{\mathrm{i}}} \cdot\left(\mathrm{L}-\text { dist }_{\mathrm{i}}\right)^{2} \cdot\left(3 \cdot \mathrm{L}-\text { dist }_{\mathrm{i}}\right) \text { if }(\text { Case }=2) \wedge(\mathrm{kL} \neq 0)
\end{aligned}
$$

Due to distributed live loads: $\delta_{\mathrm{Lwi}}:=\frac{5}{384} \cdot \frac{\mathrm{w}_{\mathrm{L}} \cdot \mathrm{L}^{4} \cdot \mathrm{g}}{\mathrm{E}_{\mathrm{c}} \cdot \mathrm{I}_{\mathrm{e}}}$

Instantaneous deflection due to service live loads: $\delta_{\mathrm{L}}:=\delta_{\mathrm{LPi}}+\delta_{\mathrm{LW}}$

Instantaneous deflection due to dead plus live loads: $\delta_{\text {total }}:=\delta_{\mathrm{D}}+\delta_{\mathrm{L}}$

Instantaneous deflection of the sustained part of live load: $\delta_{\mathrm{SL}}:=\frac{\text { Percent } \cdot \delta_{\mathrm{L}}}{100}$

Time factor for sustained loads:

$$
\begin{aligned}
& \zeta:=\| 2.0 \text { if Duration } \geq 60 \text { month } \\
& {\left[1.4+\frac{0.6}{48} \frac{(\text { Duration }-12 \cdot \text { month })}{\text { month }}\right] \text { if }(\text { Duration }>12 \cdot \text { month }) \wedge(\text { Duration }<60 \cdot \text { month })} \\
& 1.4 \text { if Duration }=12 \cdot \text { month } \\
& {\left[1.2+\frac{0.2}{6} \cdot \frac{(\text { Duration }-6 \cdot \text { month })}{\text { month }}\right] \text { if }(\text { Duration }>6 \cdot \text { month }) \wedge(\text { Duration }<12 \cdot \text { month })} \\
& 1.2 \text { if Duration }=6 \cdot \text { month } \\
& \left.\left[1.0+\frac{0.2}{3} \cdot \frac{(\text { Duration }-3 \cdot \text { month })}{\text { month }}\right]\right] \text { if }(\text { Duration }>3 \cdot \text { month }) \wedge(\text { Duration }<6 \cdot \text { month })
\end{aligned}
$$

Compression steel ratio: $\rho^{\prime}:=0$ (Since the beam is singly reinforced)

Multiplier for sustained loads: $\lambda:=\frac{\zeta}{1+50 \rho^{\prime}}$

Long-term deflection due to dead load plus sustained live load: $\delta_{\mathrm{LT}}:=\delta_{\mathrm{L}}+2 \cdot \delta_{\mathrm{D}}+\lambda \cdot \delta_{\mathrm{SL}}$

\section{Check for Crack Width:}

Ratio of distance to the neutral axis from the extreme tension concrete fiber to the distance from the neutral axis to the centroid of the tensile steel (values to be determined by the working stress method):

$\beta_{\mathrm{h}}:=1.2$

(conservative value that can be used in lieu of calculation) 
Steel stress in kips per square inch at service loads (it is permitted to use $0.6 \mathrm{f}_{\mathrm{y}}$ for normal structures):

ksi := 1000 psi $\quad \mathrm{f}_{\mathrm{s}}:=0.6 \cdot \mathrm{f}_{\mathrm{y}}$

The cover of the outermost bar measured from the extreme tension fiber to center of the closest bar: $d_{c}=3$ in

The effective tension area of concrete around the main reinforcing having the same centroid as the reinforcing)

divided by the number of bars: $A:=2 \cdot d_{c} \cdot \frac{b_{w}}{m}$

Gergely-Lutz equation for estimated crack width::

$\mathrm{w}_{\mathrm{cr}}:=\frac{\left[0.076 \beta_{\mathrm{h}} \cdot\left(\frac{\mathrm{f}_{\mathrm{s}}}{\operatorname{UnitsOf}\left(\mathrm{f}_{\mathrm{s}}\right)}\right) \cdot \sqrt[3]{\mathrm{d}_{\mathrm{c}} \cdot \mathrm{A}}\right] \cdot\left(10^{-6}\right)}{\frac{\mathrm{g} \cdot 144}{\operatorname{UnitsOf}(\mathrm{g})}}$

Allowable crack widths:

$\mathrm{w}_{\text {allowable }}:=\mid \begin{aligned} & 0.016 \text { in if Exposure = "Dry air" } \\ & 0.012 \text { in if Exposure = "Moist air, soils" } \\ & 0.007 \text { if Exposure = "Deicing chemicals" } \\ & 0.006 \text { if Exposure = "Seawater and seawater spray" } \\ & 0.004 \text { if Exposure = "Use in water retaining structures" }\end{aligned}$

crackwidth $:=\mid \begin{array}{ll}\text { "OK" } & \text { if } \mathrm{w}_{\mathrm{cr}} \leq \mathrm{w}_{\text {allowable }} \\ \text { "NG" } & \text { otherwise }\end{array}$ 